\title{
Corela
}

Cognition, représentation, langage

2-2 | 2004

Vol. $2, n^{\circ} 2$

\section{De la surface du texte à sa dimension implicite et pragmatique : lecture de deux nouvelles de Angela Carter « Master » et « Our Lady of the Massacre ».}

Catherine Paulin

\section{OpenEdition}

Journals

Édition électronique

URL : http://journals.openedition.org/corela/633

DOI : $10.4000 /$ corela.633

ISSN : 1638-573X

Éditeur

Cercle linguistique du Centre et de l'Ouest - CerLICO

Référence électronique

Catherine Paulin, «De la surface du texte à sa dimension implicite et pragmatique : lecture de deux nouvelles de Angela Carter « Master » et « Our Lady of the Massacre ». », Corela [En ligne], 2-2 | 2004, mis en ligne le 15 décembre 2004, consulté le 02 mai 2019. URL : http://journals.openedition.org/ corela/633 ; DOI : 10.4000/corela.633

Ce document a été généré automatiquement le 2 mai 2019.

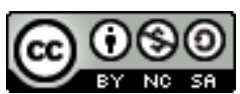

Corela - cognition, représentation, langage est mis à disposition selon les termes de la licence Creative Commons Attribution - Pas d'Utilisation Commerciale - Partage dans les Mêmes Conditions 4.0 International. 


\title{
De la surface du texte à sa dimension implicite et pragmatique : lecture de deux nouvelles de Angela Carter « Master » et « Our Lady of the Massacre $»^{1}$.
}

\author{
Catherine Paulin
}

\section{Angela Carter : bref rappel biographique. Présentation succincte des deux nouvelles.}

1 Angela Carter est née en Grande-Bretagne en mai 1940. Elle a passé son enfance dans le Yorkshire avec sa grand-mère, une femme qui appartenait à la classe ouvrière du Nord de l'Angleterre et qui semble avoir eu une grande influence sur l'évolution de sa petite-fille. Il en va de même de sa mère qui lui a transmis son goût pour la littérature et sa culture littéraire. Les références littéraires abondent dans l'œuvre de Carter, qui a aussi une fine connaissance de la théorie féministe et de la théorie littéraire de Genette et Barthes. Angela Carter se plait à analyser les structures sociétales qui lui sont familières et à démanteler leurs fonctionnements. Elle est décédée à l'âge de 51 ans d'un cancer².

Dans « Master », le personnage principal, un Anglais sans nom, auquel il est fait référence par le pronom HE, a quitté la vieille Europe pour l'Afrique, puis l'Amérique du Sud, plus précisément l'Amazonie, continents sur lesquels il joue du fusil. Décimer est pour lui le seul moyen de se sentir encore en vie : « ..., to kill became the only means that remained to him to confirm he himself was still alive, for he was not prone to instrospection and had never found any consolation in nature.", (1.63-5). Il rencontre des Indiens d'Amazonie et achète contre la roue de secours de sa jeep, une jeune fille qu'il nomme 
Friday ${ }^{3}$. Au fil du texte, la jeune fille, à qui il fait subir les pires sévices, plus encore lorsqu'il est ivre, se transforme en femme-jaguar. Elle aussi s'adonne aux plaisirs de la chasse et finit par tuer son bourreau : « His prey had shot the hunter, but now she could no longer hold the gun. », (1.277-8).

Dans " Our Lady of the Massacre ", une jeune femme, née dans la Vieille Angleterre, dans le Lancashire, orpheline dès son plus jeune âge, est contrainte de se prostituer pour survivre. Elle vole la montre en or d'un de ses clients et est envoyée avec d'autres prisonniers en Amérique, en Virginie où elle est vendue au maître d'une plantation. Le surveillant de la plantation, un dimanche après la messe, cherche à la violer: elle s'empare d'un couteau de cuisine et lui tranche les deux oreilles. Condamnée à prendre la fuite, elle trouve refuge parmi des Indiens algonkiens, qui l'adoptent. Elle épouse un des leurs. Son bonheur est grand jusqu'à ce qu'ils soient attaqués par des soldats qui menacent de tuer son bébé et de la violer. Les soldats tuent son mari, les hommes de la tribu et les dernières femmes encore en vie la tiennent pour responsable d'un tel massacre: "The squaws blamed me, how I had brought bad luck on them and cruelly repaid their kindness to me. », (1.520-1). Elle doit chercher refuge chez le pasteur dont elle devient la servante.

\section{Les paramètres énonciatifs.}

4 « Master » est un texte écrit au passé de narration (V-ED) à la $3^{\text {ème }}$ personne dans lequel il n'y a aucun passage au discours direct. Le narrateur hétérodiégétique est omniscient; le discours ne comporte que peu d'auxiliaires de modalité et la présence de l'instance narrative se fait surtout sentir à travers les choix lexicaux. L'énonciateur, ce sujet modal, n'est dans ce texte que peu présent dans le modus, il l'est bien davantage dans les choix lexicaux de dénomination et dans la structuration du dictum. Carter fait le choix d'une stratégie narrative bien différente dans "Our Lady of the Massacre » et cela, dès le titre. Le déictique OUR implique le co-énonciateur-lecteur et le sujet de l'énoncé entre d'emblée dans un rapport intersubjectif avec le lecteur qui est invité à prendre part à la structuration du récit, du dictum et du modus. Le point de vue est celui du personnage central qui établit un rapport de complicité avec le lecteur dans des incises métalinguistiques : « ... and I show the plantation a clean pair of heels, I can tell you, adding to my list of crimes the most heinous : escape from bondage" ${ }^{4}$ (1.105-6), "I am a good walker as you may judge from my trudge from Lancashire to London..." (1.107-8), " You must know that I had no knowledge of geography..." (1.116). Le récit est le plus souvent à la 1ère personne au passé de narration. Certains passages sont cependant au présent de narration dans le but de rapprocher le temps de l'événement du temps de la lecture, comme s'il s'agissait d'une histoire bien connue; les reports de paroles au discours direct ne sont pas rares. Dans un but de réalisme qu'elle veut totalement illusoire, Carter reproduit certaines idiosyncrasies de la parole du personnage central dans le discours de la narratrice intradiégétique : “..., my father a poor farm servant, and me mam and he both died of plague when I was a little thing so me and me brothers and sisters left living were put on the parish...", (1.6-8).

5 Le texte de "Master" est dominé par un énonciateur qui pratique l'effacement des marques de sa présence afin de conférer au texte une fonction descriptive. L'énonciateur, qui n'implique pas le co-énonciateur, prend en charge l'histoire qu'il raconte dans des énoncés dans lesquels s'exprime sa conception de la situation. Le point de vue n'est donc 
pas externe ainsi qu'en témoignent les choix lexicaux et certains connecteurs, comme nous le découvrirons plus loin. L'objectivation du récit dans "Our Lady of the Massacre" passe par la mise en place d'un co-énonciateur universel. Le narrateur-énonciateur intradiégétique est à la source du point de vue exprimé dans les contenus propositionnels.

J'appelle 'énonciateurs' ces êtres qui sont censés s'exprimer à travers l'énonciation, sans pour autant qu'on leur attribue des mots précis; s'ils 'parlent', c'est seulement en ce sens que l'énonciation est vue comme exprimant leur point de vue, leur position, leur attitude, mais non pas, au sens matériel du terme leurs paroles. (Ducrot 1984 : 204)

Les deux textes rassemblent des contenus propositionnels ayant un fort degré de cohésion thématique et une visée commune: la démystification et le renversement de préconstruits culturels. Les sujets, contradictoires, victimes et rebelles, sont en quête d'identité. Ces textes sont empreints d'intertextualité; le discours se construit par rapport à d'autres discours qu'il subvertit ${ }^{5}$. Les sujets se font en déconstruisant un discours sur le monde qui s'impose à eux. Il en résulte des textes dont l'interprétation n'est guère opérée en fonction de la situation. En effet, la situation dans laquelle se trouvent les personnages est à la fois banale et magique, totalement irréelle, puisqu'il convient de "défamiliariser" le réel afin de rentrer dans un univers qui tient du conte, dans lequel le particulier importe peu. Le texte est concentré, il vise à démystifier des stéréotypes, les mots "clignotent", les repères entre animalité, humanité; vie, mort; masculin, féminin sont brouillés. Carter est bien connue pour faire un usage intensif d'un intertexte qu'elle cherche à démystifier. Cependant :

..., comme on doit s'y attendre, il n'y a pas de désacralisation qui n'aille sans une certaine fidélité à ce à quoi il va être porté atteinte. Ce qui veut dire que le modèle doit rester apparent dans sa recréation critique. (Pedot $2002: 103$ )

7 Le rôle du linguiste-analyste est de rendre compte des effets de sens produit par le texte, que celui-ci soit empreint d'intertextualité, de métatextualité ou non.

\section{Loin du réalisme social : mélange du narratif et du poétique et brouillage de la frontière entre le réel et l'irréel.}

Dans ces deux textes, Carter défend les opprimés, que ce soit les indigènes d'Amazonie asservis par les représentants de valeurs coloniales en pleine dégénérescence ou les prolétaires de la Vieille Angleterre réduits au vol, à la prostitution ou à la servitude par les représentants des valeurs bourgeoises ou de celles du clergé. Ces textes ont bel et bien une teneur moralisante et représentent un monde binaire composé des dominants et des dominés. Jusque-là, on pourrait croire qu'il s'agit d'une critique sociale sur un ton réaliste. Or, il n'en est rien puisque, dans l'univers de ces nouvelles, tout est dans tout: Carter opère une série de renversements qui font des victimes des bourreaux et viceversa. L'inversion des rôles et la révolte du dominé est un motif cher à Carter qui passe par une écriture poétique et dramatique, loin du réalisme social. Carter ouvre la fable "Vampirella" par ces mots : "Can a bird sing only the song it knows or can it learn a new song?" . Les personnages se construisent et se déconstruisent dans un espace narratif peu stable. Le figural, les comparaisons, les métaphores et les métonymies permettent la mise en place de ces renversements multiples, confèrent un caractère hyperbolique aux textes et 
jouent un rôle prépondérant dans les glissements qui s'opèrent entre humanité animalité - réification; nature - culture; verbal - pictural; victime - rebelle - bourreau...

Dans "Master", He, privé de nom propre, est représentatif d'une catégorie d'hommes. Les animaux sont eux aussi stéréotypiques, ce qui apparaît bien dans le GN qui suit: (1.27)“ he decimated herds of giraffe and gazelle" dans lequel le fonctionnement en collectif des noms "giraffe" et "gazelle" sans marque de pluriel renvoie qualitativement à l'espèce. L'emploi de THE + N-s dans les GN suivants, (1.32)"the silken indifference of the great cats " et(l.33)"he developed a speciality in the extermination of the printed beasts, leopards and lynxes"permet de renvoyer à une classe d'animaux qui est définie en extension (il s'agit de tous les éléments dont se compose la classe), par contraste avec d'autres espèces. Le règne humain est privé du soutien des Dieux contrairement au règne animal doté d'un langage idéographique : (1.33-7) “... the printed beasts, leopards and lynxes, who carry ideograms of death in the clotted language pressed in brown ink upon their pelts by the fingertips of mute gods who do not acknowledge any divinity in humanity. ${ }^{6}$ "En proposant un repas de viande à Friday, il lui propose la chair interdite et court à sa propre perte. Tandis que les félins ont un langage écrit, la bête humaine est réifiée : (1.3-5) “... until, in time, the insatiable suns of Africa eroded the pupils of his eyes, bleached his hair and tanned his skin until he no longer looked the thing he had been but its systematic negative". Le groupe nominal complexe "the thing he had been" est composé d'une tête "thing" et d'une expansion propositionnelle qui est une relative définitoire. L'ensemble est attribut du sujet "he" du fait du sémantisme du verbe copule "look". Carter a choisi de déshumaniser le sujet grâce à "thing" en faisant fi de l'antinomie entre humain et nonhumain. Le nom "thing", archilexème par excellence, permet la co-existence d'un degré maximal d'indétermination et d'une forme de surdétermination, de par la métaphore réifiante et l'ajout de la relative définitoire. Carter aurait pu adopter une toute autre stratégie pour poser la différence entre l'identité du personnage auparavant et maintenant: "he no longer looked what he had looked like", "he no longer looked the way he had looked". De plus, le sujet thématique "he"est totalement désagentivé, nonintentionnel. Il est l'objet anonyme de la perception d'un énonciateur-narrateur distancié. Le prédateur qu'il est devenu est absent du récit de son histoire et est présenté comme un autre que lui-même aux lignes 24-6: “ ... if little of him now pertained to the human, the eyes of his self still watched him so that he was able to applaud his own depredations". Le nom "self" renvoie bien au moi conscient, il devient son propre objet de perception, ce qui est marqué par le pronom "him" qui porte la marque de l'accusatif. Le travail fait ici n'est-il pas un travail de traduction, d'anamnèse des perceptions et des peurs inconscientes? Le groupe nominal "his own depredations" est ambigu : s'agit-il des dégradations physiques et morales qu'il endure ou des dommages qu'il fait subir à autrui, à la nature ou encore des deux? Il est également représenté comme une bête féroce : (1.20) "his ferocity", (1.87) "licking his chops" tandis qu'elle est sauvagement féline : (1.105) "she had the immovable smile of a cat", (1.244) "her pelt". Le meurtre et le fantôme de la mort $\mathrm{du}$ prédateur règnent sur tout le texte dont il convient de souligner l'orientation téléologique.

Dès la ligne 6, il est question de son exil qui est représenté comme "l'imitation de la mort", "un deuil programmé" : "...; he became the white hunter, victim of an exile which is the imitation of death, a willed bereavement...". L'autre victime, extérieure à lui-même devient rebelle et la voix narrative le rend responsable du meurtre annoncé, (l.196-197): "... since he had taught her to eat meat, now she must be death's apprentice". Le 
connecteur SINCE est polysémique et indique le point d'origine, temporel ou causal. Ici, les deux valeurs sont confondues : la fin est annoncée dès le premier repas de viande. La structure corrélative de la ligne 231 "As she grew more like him, so she began to resent him", annoncée aux lignes 182 et 194 par “... as she had seen Master do"montre leur évolution parallèle mais liée. AS cataphorique est un adverbe corrélatif de degré qui marque l'accroissement parallèle repris par SO qui a une double orientation anaphorique et cataphorique. Cette construction corrélative ne rentre ni dans le cadre de la subordination ni dans le cadre de la coordination: aucune des deux propositions ne saurait être effacée sans nuire à la grammaticalité de l'énoncé; les propositions ne sauraient être inversée ${ }^{7}$. Les deux propositions se déterminent l'une l'autre - le degré de p1 est identifié au moyen de la construction en AS... SO au degré de p2 - comme les deux protagonistes se déterminent l'un l'autre. Dans ce texte allégorique et moral, la pensée binaire s'effondre : la victime et le bourreau sont-ils encore dissociables ? Dès le début, le prédateur, qui est déjà mort et étranger à lui-même ${ }^{8}$, est représenté comme sa propre victime; il met en scène sa propre mort en initiant sa proie à son art. Quelques lignes avant la fin du texte, ligne 277, on lit: "His prey had shot the hunter". S'il n'est repéré que par rapport à lui-même et à la situation (THE), la proie est repérée par rapport au prédateur grâce au possessif HIS. Il a engendré sa victime et l'auteur de sa mort. La victime libérée par la mort du chasseur ne pourra plus tenir de fusil, tandis que la dépouille du chasseur pourrira loin de chez lui, lignes 282-3: "Then only the flies crawling on his body were alive and he was far away from home". Cet énoncé, à tonalité ironique, sur lequel se termine le texte est ambigu: faut-il le plaindre car il est mort inutilement ou faut-il entendre un appel sentimental mais ironique qui ferait un dernier écho à l'expression "ambiguous couple" de la ligne 133 et ferait de la jeune indienne une proie bien ingrate?

\section{Dénomination et métamorphoses dans “Our Lady of the Massacre".}

11 La nouvelle s'ouvre sur la question du nom du personnage principal :

"My name is neither here nor there since I used several in the old World that I may not speak of now; then there is my, as it were, wilderness name, that now I never speak of ..." (1.1-3).

Le lien entre la conscience que l'on a d'être soi et le lieu et l'époque où l'on vit est immédiatement posé. Les changements identitaires - contingents aux conditions dans lesquelles elle évoluait - que Mary a traversés font qu'un seul nom ne lui suffit pas et qu'il ne renvoie qu'à celle qu'elle est ou a été dans un ancrage situationnel et culturel particulier et non à la multiplicité de ses "moi" qui fait d'elle ce qu'elle est. Le nom qu'elle porte au moment du récit - rétrospectif - n'est pas significatif de son identité : “... what I call myself in this place, therefore my name is no clue as to my person nor my life as to my nature.", (1.3-5). La jeune fille de ferme qui s'occupe de la traite, the 'Lancashire milkmaid' (1.68), devient rapidement à Londres la putain du Lancashire, the 'Lancashire whore' (1.68). Il semble que le lien métonymique entre elle et la fonction qu'elle exerce dans la société dans laquelle elle se trouve est tel que les deux sont indissociables. Le déterminisme social et le regard de l'autre sont tels qu'elle n'existe qu'à travers ce qu'elle fait. Suite au vol de la montre en or d'un de ses clients, elle est déportée en Virginie. Là, son maître lui porte un intérêt particulier bien qu'elle n'ait que dix sept ans et décide que 
plutôt que de travailler dans la plantation de tabac, elle lui servira de cuisinière. L'intendant qui connaît son histoire lui refuse toute possibilité de changement : “... since I had been a whore in Cheapside, I should not play the honest maid in Virginia" (1.86-7). En dépit du changement de continent et de la peine à laquelle elle est condamnée, les conditions de sa déportation d'un monde à un autre, mais dans le maintien d'un code moral unique, font qu'elle est perçue comme la même alors qu'elle souhaite devenir cette autre qui est en elle. Dans le texte, la seule personne qui l'appelle par son nom, ou plutôt un de ses noms, Sal - diminutif de Sally - en discours direct est le jardinier, un esclave noir que le narrateur réduit à une représentation stéréotypique et ironique du "bon esclave" : " a good-natured kind of Negro man and a slave" (1. 98). Le prémodifieur KIND OF dans le groupe nominal complexe GN1 OF GN2 fait de lui une espèce d'homme, un nègre, pas vraiment un homme. Il est identifié comme "sous-homme" et esclave grâce au déterminant A qui renvoie à une extraction d'une occurrence de la classe des occurrences.

13 La marque au fer rouge qu'elle porte au poignet est d'après elle reconnue comme telle par l'Indienne qu'elle rencontre et qui deviendra sa mère d'adoption. Dans ce passage en focalisation interne, elle ne parvient pas à se défaire de ses propres préconstruits culturels et perçoit l'altérité à travers le prisme de sa propre culture et de sa propre histoire :

"This woman sees the brand on my hand and grunts to herself, as though she knows the meaning of it and will not fear me for it, or, rather, does not fear me because of it, but, all the same, does not like the look of me." (l.168-171).

Deux remarques: la première à propos du connecteur AS THOUGH qui présente le contenu propositionnel de la subordonnée sous l'angle de la non actualisation potentielle; la seconde, relative à l'emploi du présent simple "knows" là où on s'attendrait à un prétérit modal contrefactuel "knew" puisque nous sommes dans l'irréel absolu et que cette femme indienne n'a pas été en contact avec des hommes ou des femmes de race blanche auparavant ${ }^{9}$. Il y a tension entre THOUH marqueur de concessivité qui présente le contenu de p2 comme non-congruent tandis que le présent rapproche l'irréel de l'actuel. Immédiatement, au contact de cette Indienne qu'elle trouve belle ${ }^{10}$, Mary opère une métamorphose physique, se défait de ses vêtements, signes de son appartenance culturelle et s'habille en Indienne. Très vite, puisque comme les autres femmes elle travaille dans les champs, elle devient aussi brune que ses hôtes. Seul le bleu de ses yeux trahit son appartenance ethnique. La jeune femme, empreinte de la perception que les siens ont d'elle, doute de sa capacité à être une bonne épouse pour Tall Hickory qui est la traduction anglaise du nom qu'il porte et qui signifie "grand noyer blanc d'Amérique" : 'How can I be the good man's wife, mother, for I was a bad woman in my own country.'(1.295-6). Confrontée à ce qu'elle perçoit comme pureté et honnêteté de la part des Indiens, elle est animée par un sentiment de culpabilité et se convainc même d'avoir volé par vocation et non par besoin : “... I was a thief by natural vocation"(1.299-300). De ce fait, elle dénie ses propos et relaie l'opinion de ceux qui l'ont condamnée, ligne 59 et ligne 72 : “...want made a thief of me". Bien qu'elle se considère comme intégrée au groupe des femmes, “...we squaws...”(1.349) et à sa nouvelle culture, elle ne donne pas à connaître au lecteur co-énonciateur le nom indien qu'on lui a donné et ne semble exister que comme épouse de Tall Hickory ou mère de Little Shooting Star (1.386-7). Comme suite au massacre des Indiens par les soldats anglais et au rejet dont elle fait l'objet, y compris de la part de sa mère d'adoption ${ }^{11}$, elle est recueillie par un pasteur et sa femme à qui elle se présente sous le nom de Mary, d'après le nom de la vieille femme qui l'avait recueillie 
après la mort de ses parents, dans le Lancashire. De ce fait, elle se sent désincarnée et étrangère à elle-même: "And when they ask my name, I give "em the name of my old Lancashire lady, which is Mary, and stick by it, so I live on as if I were a ghost." (1.530-2). Little Shooting Star est, lui aussi, condamné à porter une marque distinctive - une cicatrice au front, suite à la blessure qui lui a été infligée par les soldats - et à avoir une double identité : le nom chrétien que lui donne le pasteur et que sa mère refuse de lui donner chaque fois qu'ils sont seuls, et le nom indien de sa naissance que sa mère affectionne.

L'absence, le "manque" semble être un élément central qui explique la douleur et l'absence à elle-même du personnage principal. La mise en oeuvre de ce vide semble être la suivante : en ayant plusieurs noms selon les lieux et les circonstances, elle finit par ne plus répondre à aucun. De surcroît, alors que, parmi les Indiens, elle se comporte comme un être digne de confiance, sa mère d'adoption qui avait reconnu sa valeur et lui avait accordé sa confiance, se fiant soudain aux apparences trompeuses, la lui retire - et c'est un renversement. On assiste à une ultime inversion et cela bien que Mary se soit engagée, dans un passage au discours direct, à l'honnêteté qu'il ne faut pas entendre comme matérielle uniquement: "So neither desire nor want can make a thief of me, here." (1.314). "Want" appartient à la classe du verbe comme à la classe du nom et est ici employé comme nom: il s'agit pour la locutrice de pondérer les effets du manque en prenant en compte la relation qui existe entre elle et son peuple d'adoption. Elle exprime le manque en terme de désir ou de besoin. Le recours au nom "want" semble correspondre à une vision euphémique de tous les manques dont elle fait l'expérience. "Want", qui exprime de manière édulcorée ce que "lack"12dénote, renvoie à l'absence qui "sanctionne de manière programmatique la vacuité d'un intérieur pris comme repère." (Delmas 1998 : 144). Or, dans le co-texte ci-dessus, l'objet du manque n'est pas exprimé : faut-il entendre absence / manque de biens matériels ou, de façon plus implicite, manque à soi ? Cette vacuité s'oppose à une visée préconstruite : il y a absence de complément et absence de construction identitaire du personnage qui s'en remet à la situation. Le thème de l'exil voulu ou subi est présent aussi bien dans "Master"que dans "Our Lady of the Massacre" et est à rapprocher de celui du manque ou de l'absence. Le pays d'origine des protagonistes, la Vieille Angleterre est hors situation mais néanmoins présent dans les préconstruits culturels qui sont les leurs, soit à l'égard de l'Autre, soit envers eux-mêmes ou leur propre culture dont ils relient les principes doxiques en les renversant. Se définir a contrario, c'est encore se définir par rapport à : il est difficile de "penser la différence sans la subordonner à l'identité" (Felman 1992 : 142). La déstabilisation des codes, les renversements et les inversions débouchent sur des métamorphoses du moi désenchanté, désabusé et perdu des deux personnages. En ce sens, ces textes sont dérangeants.

Dans "Master", l'inversion est totale : la victime et la rebelle sont rassemblés en un seul personnage; le féminin prend le pas sur le masculin. Si le féminin est également associé à la mort dans "Our Lady of the Massacre", la question du rapport entre les sexes est déplacée sur celle de l'identité culturelle au féminin qui est occultée dans la culture par le masculin. La dimension parodique et ironique du texte donne à voir le pouvoir destructeur de l'homme ainsi que celui des constructions mythiques et phantasmées quant à la culture de l'Autre. Le texte, à visée éthique, dénonce cette dérive; citons quelques exemples dans lesquels le personnage central s'engouffre dans la forêt des représentations mythiques que le texte débusque. Il s'agit de commentaires dans des incises ou passages à valeur métalinguistique : “...., for these savages are not half so free with their feelings as we are,..." (l.183), "They were cooking what they call succotash, a 
great pot on an open fire and a naked savage squatting before it, calm as you please, fanning the flames with a birchbark fan." (1.201-3), "How do they live, these so-called demi-devils?" (1.238). Mary s'inscrit contre les représentations de l'Indianité des hommes d'Eglise et si elle déconstruit le religieux, elle accepte les "bonnes valeurs": “...; the Indians had damned me for a good woman once and for all."(1.566-7).Notons que dans cet énoncé, le thème est constitué du sujet agentif "the Indians" et que Mary est argument objet du verbe, ce qui fait d'elle un sujet expérient mais passif. Notons aussi la dimension subversive sous-jacente de l'oxymore qui réunit dans une même prédication les termes "damned" et "good".

\section{Langage et pouvoir.}

Dans "Master", les hommes n'ont pas la parole et sont privés de toute possibilité de sauver la part d'humanité qui les habite. HE est accablé par le poids du silence de la forêt amazonienne : "A green, irrevocable silence closed upon him in that serene kingdom of giant vegetables" (1.46-7) tandis qu'elle se rapproche de plus en plus de cette animalité qu'à eux deux ils déciment. Si les humains sont privés de langage articulé : "She could no longer twist her scarlet tongue around the syllables of his name, 'mas-tuh'; when she tried to speak, only a diffuse and rumbling purr shivered the muscles of her throat..." (1.248-50), les cris et les rires font sens et permettent à la jeune Indienne d'expulser ses émotions, sa joie lorsqu'elle abat une proie ou sa douleur lorsqu'elle fait l'objet de violences sexuelles répétées : "Her screams were a universal language; even the monkeys understood she suffered when Master took his pleasure, yet he did not." (1.229-32). Le langage iconographique sur le pelage des félins (1.33-7) ou sur le corps des Indiens sont chargés d'une signification qui reste muette.

Dans cette nouvelle, il est difficile de décider quel est le personnage privilégié qui est au centre du texte. Le lecteur est guidé dans une lecture empathique à partir de deux centres qui se confondent pour finalement se dissocier totalement par la mort de l'un d'eux. Il n'est pas donné au lecteur d'accéder à la psyché ni de l'un ni de l'autre des deux personnages qui n'ont pas de "réalité psychologique". La parole morte des personnages étrangers à eux-mêmes reprend vie dans la lecture interprétative par laquelle s'établit une relation forte entre la nouvelliste et le lecteur qui est en décalage permanent avec les personnages et qui, à partir des agencements du texte, construit le message. Ce texte, dont l'orientation téléologique est à souligner, n'est cependant pas univoque: les frontières tombent, entre le dehors et le dedans, entre les sexes, entre les espèces, entre les rôles...

... le "dehors" est à la fois un "objet de connaissance" (ce qui est matériellement observable) et ( une sorte de ) vision ("le dedans lui-même vu du dehors). Cette sorte de vision tient au fait que le "dehors" signifie toujours quelque chose pour le personnage et le lecteur. Cette sursignification explique sans doute le passage de l'objet de connaissance à la vision, dans la mesure où la sursignification n'a de pertinence propre que pour un sujet donné.

Dès lors, en raison de cette sursignification même, la vision "du dehors" n'est pas une vision indépendante : elle ne peut littéralement pas avoir l'autonomie des deux types de vision précédents (la vision avec et la vision du dedans), car, seule, elle resterait confinée dans, sinon l'insignifiance, du moins l'inintelligible, du point de vue des personnages comme de celui des lecteurs, tous deux confrontés à "l'apparition de rien pour personne"(Rabatel 1997 : 31) 

Par des biais énonciatifs différents, la dimension idéologique implicite semble bien être l'essentiel de cette écriture fictionnelle narrative caractérisée par l'unité de son histoire et par sa forte teneur téléologique. Dans "Master", la narration est en V-ED et à la 3ème personne, ce qui crée un effet de distanciation compensé par la dimension quasi-picturale de certaines scènes. Dans "Our Lady of the Massacre", la narration est tantôt en V-S tantôt en V-ED, à la 1ère personne, ce qui, jusqu'à un certain point, crèe une identité de vision entre le personnage réflecteur et le lecteur à qui on donne l'illusion d'entendre le discours oral du personnage central. Dans les deux cas, le texte narratif est là pour dire, entre dire et ne pas dire, de l'idéologique, pour s'engager politiquement contre le colonialisme, spirituellement contre le religieux. Il s'agit là de textes impressionnistes et d'une écriture de l'ambigu. Le lecteur, qui est mis en situation est "impressionné" par l'histoire et est amené à donner une valeur ajoutée au texte en raison de sa dimension implicite. Le titre joue un rôle majeur et éclaire non pas le sens mais un sens de ces textes plurivoques. Les attentes suggérées par le titre sont bouleversées dans une série de 
renversements; il suffit pour s'en apercevoir de confronter le titre "Master" et la dernière phrase de la nouvelle ou encore de voir que la pluralité des interprétations du titre "Our Lady of the Massacre" n'en fournit aucune qui traduise le message qui se dégage du texte. Le discours qui se tient est sans cesse traversé par l'autre, cet autre qu'est le lecteur, cet extérieur intériorisé qu'est le métatexte. L'absence de dénomination des personnages ou la pluralité de noms contribuent, ainsi que la fluctuation de l'instance narrative, à repousser le lecteur qui demeure cependant au coeur du texte.

\section{BIBLIOGRAPHIE}

ADAM, J-M. (1992) Les textes : types et prototypes, Paris, Nathan.

ADAM, J-M. (1999) Linguistique textuelle. Des genres de discours aux textes, Paris, Nathan.

ANSCOMBRE, J.C. et DUCROT, O. (1983) L'argumentation dans la langue, Bruxelles-Liège, P. Mardaga eds.

ARMENGAUD, F. (1991) La pragmatique, Paris, PUF.

AUSTIN, J.L. (1962) How to Do Things with Words, Oxford University Press.

BERRENDONNER, A. (1981) Eléménts de pragmatique linguistique, Paris, Editions de Minuit.

BENVENISTE, E. (1966) Problèmes de linguistique générale,1, Paris, Gallimard.

BENVENISTE, E. (1974) Problèmes de linguistique générale, 2, Paris, Gallimard.

CARTER, R. \& SIMPSON, P. (1989) Language, Discourse and Literature, London and New York, Routledge.

CULIOLI, A. (1990) “Sur le concept de notion”, in Pour une linguistique de l'énonciation, Gap, Ophrys.

CULIOLI, A. (1990) “La Négation : marqueurs et opérations”, in Pour une linguistique de l'énonciation, 1, Gap, Ophrys. 91-113.

DANON-BOILEAU, L. (1982) Produire le fictif, Paris, Klincksieck.

DANON-BOILEAU, L. (1987) Le sujet de l'énonciation, Psychanalyse et Linguistique, Gap, Ophrys.

DANON-BOILEAU, L. (1995) Du texte littéraire à l'acte de fiction : lectures linguistiques et réflexions psychanalytiques, Gap, Ophrys.

DELMAS, C. (1998) « Lexique et grammaire du manque en anglais » in Recherches en Linguistique Etrangère XIX, Mélanges pour Jean Tournier, Textes réunis par Catherine Paulin, Annales littéraires de l'Université de Franche-Comté.

DUCROT, O. (1980) Les mots du discours, Paris, éditions de Minuit.

DUCROT, O. (1984) Le dire et le dit, Paris, Minuit.

DUCROT, O. (1991) Dire et ne pas dire, Principes de sémantique linguistique, Paris, Hermann.

FELMAN, S. (1992) La folie et la chose littéraire, Paris, Editions du Seuil.

KERBRAT-ORECCHIONI, C. (1980) L'énonciation de la subjectivité dans le langage, Paris, Armand Colin. 
KERBRAT-ORECCHIONI, C. (1986) L'implicite, Paris, Armand Colin.

LEHMANN, A. \& MARTIN-BERTHET F. (1998) Introduction à la lexicologie, Sémantique et morphologie, Paris, Dunod.

LOUVEL, L. (ed). (2002) La Nouvelle Anglo-Saxonne Contemporaine. Réflexions sur un genre, Poitiers, La Licorne.

OGDEN, C.K. and RICHARDS, I.A. (1923) The Meaning of Meaning, London, Routledge and Kegan.

MAINGUENEAU, D. (1976) Initiation aux méthodes de l'analyse du discours, Paris, Hachette.

MAINGUENEAU, D. (1987) Nouvelles tendances en analyse du discours, Paris, Hachette.

MAINGUENEAU, D. (1993) Eléments de linguistique pour le texte littéraire, Paris, Dunod.

MAINGUENEAU, D. (1994) L'Enonciation en linguistique française, Paris, Hachette.

MORTUREUX, M-F. (1997) La lexicologie entre langue et discours, Paris, Sedes.

PAULIN, C. (2001) "Interénonciativité dans "A Lady of Letters" et "Soldiering On" de Alan Bennett, dans Talking Heads, BBC Books, Actes du Colloque organisé par le Centre de Recherches Image/Texte/Langage "Le destinataire dans les écrits intimes", Centre Image/Texte/Langage, Editions Universitaires de Dijon.

PAULIN, C. (2002) "Lecture linguistique de Faces in the Water de Janet Frame : et si le texte était à la folie ce que l'extra - lucidité est au délire ? “, Annales de l'Université de Franche - Comté, série ALSO (Art, Littérature et Société).

PAULIN, C. (A paraître), “Ecrits introspectifs et relations intersubjectives : le récit autobiographique de la thérapie de Sylvia Plath (décembre 1958 - mars 1959), Actes du Colloque organisé par AORISTE (Atelier Orléanais de Recherche Interdisciplinaire sur les Textes Ecartés), Université d'Orléans.

PAULIN, C. (2004), « Lexique, syntaxe, langue et créativité dans The God of Small Things », Recherches en Linguistique Etrangère, $\mathrm{n}^{\circ} 23$.

PAULIN, C. (A paraître), «L'implicite dans « Swans » de Janet Frame, New Zealand, 1951, CRILA, Pôle Nouvelle, Presses Universitaires de Rennes.

PEDOT, R. (2002) « Enchantements et désenchantements de la (re)création littéraire dans « The Bloody Chamber » (Angela Carter) in La Nouvelle Anglo-Saxonne Contemporaine. Réflexions sur un genre, Poitiers, La Licorne.

SEARLE, J. R. (1972) Speech Acts, Cambridge University Press.

RABATEL, A. (1997) Une histoire du point de vue, Paris, Klincksieck.

RIVARA, R. (2000) La langue du récit. Introduction à la narratologie énonciative. Paris,L'Harmattan.

RIVARA, R. (2004) Pragmatique et énonciation, Publications de l'Université de Provence.

\section{NOTES}

1. In Burning your Boats (Collected Short Stories) by Angela Carter, UK H/B, Chatto \& Windus, 1995. «Master » se trouve dans le livre Fireworks: Nine Profane Pieces, 1974 et «Our Lady of the Massacre » dans Black Venus, 1985.

2. Margaret Atwood écrit dans The Observer Newspaper, une semaine après sa mort: She was the opposite of parochial. Nothing, for her, was outside the pale: she wanted to know about everything and 
everyone, and every place and every word. She relished life and language hugely, and revelled in the diverse. http://perso.wanadoo.fr/andrew.milne/biography.htm

3. Il s'agit d'une allusion à Robinson Crusoe.

4. Le soulignement est le nôtre ; tel sera le cas dans les citations suivantes.

5. Carter convoque et révoque des textes du canon littéraire, en particulier ici, les textes de Daniel Defoe : Robinson Crusoe dans " Master », Moll Flanders dans " Our Lady of the Massacre ». Moll Flanders comme Robinson Crusoe ont un statut mythique. Moll Flanders est une fable qui met en scène le vol, la prostitution, le crime et le châtiment et dans laquelle l'héroïne, Moll, qui raconte sa propre histoire, finit, après bien des tribulations, par se repentir de ses péchés et par faire le bien tant sur un plan moral qu'économique.

6. La polysémie de l'adjectif «clotted» (que l'on peut considérer soit comme un participe passé adjectivé soit comme un nom suffixé + - ed, qui signifie «muni de ») est intéressante : le verbe "clot» est dérivé par conversion du nom «clot» qui signifie «caillot, embolie» et par métaphore «balourd, empoté ». Faut-il entendre que l'écriture divine est maladroite, que c'est une condamnation pour les félins à être décimés par la race humaine?

7. Les subordonnées adverbiales sont a priori effaçables. Les propositions coordonnées peuvent être inversées.

8. "Master", (1. 63-5), cf; p. 1, note $2:$ «... to kill became the only means that remained to him to confirm he himself was still alive, for he was not prone to instrospection and had never found any consolation in nature ".

9. Sa réaction devant l'apparence physique de l'étrangère le prouve ainsi qu'une remarque émanant de Sally elle-même : "...where no person of my country had ever trod before. ", (l. 161-2).

10. «But I am struck by her looks, she is a handsome woman, not red, but wondrous brown... » (1.172-3).

11. "'You unkind daughter!' For she thought I was hastening to cast my lot in with the English, which was not so, by any means." (1.474-6).

12. . Avec «lack», elle mettrait son destinataire en demeure "d'évaluer de manière situationnellement spécifique l'ajustement de la prédication au cas évoqué » (DELMAS 1998 : 144). Avec «lack», on aurait une stratégie quasi-modale et il faudrait que l'objet du manque soit instancié. Elle trouverait alors inacceptable le manque de $\mathrm{x}$ et l'énoncé s'inscrirait clairement dans le domaine du jugement appréciatif.

13. " 'Now, my dear', says my husband, who was stone-cold sober because he'd a mortal terror of the edge of my tongue. 'I must ask you to talk to this fellow in your own language » (1.428-30).

\section{RÉSUMÉS}

Grâce à l'analyse de faits de langue et des dispositifs d'énonciation, cet article vise, dans une perspective sémiotique, à éclairer le rapport entre modes de représentation et production du sens par le lecteur. Il s'agit de passer de la microanalyse à la dimension plus globale de textes, à forte teneur idéologique, qui offrent une représentation du pouvoir du langage, du rapport entre identité et dénomination, masculin et féminin, dominant et dominé dans une langue saturée.

This paper, which provides an analysis of both discursive evidence and enunciative parameters, aims at shedding light on the relationship between modes of representation and the production 
of meaning by the reader. The author tries to bridge the gap between microanalysis of specific data - lexical, grammatical or syntactic - and a more global approach of ideologically loaded texts, which offer a representation of the power of language, the relationship between identity and denomination, the masculine and the feminine, the master and the slave, the rich and the poor in a saturated language.

\section{AUTEUR}

\section{CATHERINE PAULIN}

Université de Franche-Comté. 